\section{Molecular characterization of Methicillin-resistant Staphylococcus aureus isolated from the pig production chain in Northern Italy}

\author{
Ancuta Cezara Simon, ${ }^{1}$ \\ Valentina Baldo, ${ }^{2}$ Nadia Losio, ${ }^{2}$ \\ Virginia Filipello, ${ }^{2}$ Angelo Colagiorgi, ${ }^{1}$ \\ Federico Scali, ${ }^{2}$ Emanuela Zanardi, ${ }^{1}$ \\ Sergio Ghidini, ${ }^{1}$ Adriana Ianieri, ${ }^{1}$ \\ Giovanni Loris Alborali ${ }^{2}$ \\ ${ }^{1}$ Department of Food and Drug, \\ University of Parma; ${ }^{2}$ Lombardy and \\ Emilia Romagna Experimental \\ Zootechnic Institute (IZSLER), Brescia \\ Section, Italy
}

\begin{abstract}
This study aimed to evaluate the molecular characteristics of methicillin resistant Staphylococcus aureus (MRSA) isolated in the swine chain in Northern Italy. A sample of 50 fattening units located in Lombardy was selected. Five cutaneous samples at slaughtering and three environmental samples at farm were collected from each unit giving a total of 250 and 150 samples, respectively. A total of 25 MRSA isolates were isolated from 400 samples, in 17 different fattening units. At farm, 12 out of 250 samples were positive for MRSA $(4,8 \%)$, and 13 out of 150 samples at slaughter were identified as MRSA $(8,7 \%)$, giving an overall incidence among samples of $6,25 \%(\mathrm{n}=25)$. Molecular characterization was carried out using multi-locus sequence typing (MLST) and spa-typing. Outcomes showed that most of the isolates belonged to ST398, carrying spa-types t899, t011, t18498, t1939, t1200, and t304. Nonetheless, three isolates were identified as ST97 (t1730 and t4795), and one as ST30, showing spa-type t318. Furthermore, a novel ST was identified, namely 5422, showing spa-type t1730. Heterogeneity in genotypes within the same farm was also found in different fattening units, with concern for the possibility of the exchange of genetic determinants among different lineages. Genetic diversity among MRSA isolates in pig fattening units has been observed, highlighting the possibility that some isolates could be able to infect different hosts, including human.
\end{abstract}

\section{Introduction}

Methicillin-resistant

Staphylococcus aureus (MRSA) is the most commonly identified multidrug resistant pathogen in many parts of the world (Taylor, 2013). The capability of Staphylococcus aureus strains to easily adapt to the selective pressure of antimicrobials made it became a threat to public health. In fact, due to the extensive use of methicillin in clinical settings, $S$. aureus evolved and acquired the resistance to this antimicrobial. Resistance to methicillin in $S$. aureus is mediated by the $m e c \mathrm{~A}$ gene and its homologue, $m e c \mathrm{C}$, which are chromosomally located on the mobile genetic element staphylococcal cassette chromosome (SSCmec). The SSCmec codes for a penicillin binding protein (PBP) $2 \mathrm{a}$, with a low affinity for beta-lactams. (Abdelbary, Basset, Blanc, \& Feil, 2017).

Initially, MRSA emerged in healthcare settings (Hospital-acquired, HA-MRSA), but more recently it became also able to colonize humans outside hospitals (communityacquired, CA-MRSA) and animals (Livestock-associated, LA-MRSA) (Zarazaga et al., 2017). In the case of foodproducing animals, a specific clone (CC398) has been found in several countries, including Austria, Belgium, Canada, Denmark, France, Germany, The Netherlands and Italy (Oniciuc et al., 2017; Sieber et al., 2018).

Pig herds are an important reservoir for MRSA CC398, that has been frequently found also in cattle and poultry (Lassok and Tenhagen, 2013). In pig farms, it has been reported that dust particles and air systems are possible factors for MRSA transmission (Doulgeraki et al., 2016). Although CC398 has been found to colonize animals, only few isolated cases of clinical infections in animals have been reported (Aires-de-Sousa, 2017). MRSA can also occur in slaughterhouses by entering in or on animals, where it can become part of the resident microbiota (Van den Broek et al., 2009). Furthermore, LA-MRSA clones have been found in raw meat products intended for human consumption (Petternel et al., 2014; Zehra et al., 2019).

The aim of this study was to investigate the molecular characteristics of MRSA isolated in pig fattening units in Northern Italy, through multi-locus sequence typing (MLST) and staphylococcal protein A typing (spa-typing).

\section{Materials and Methods}

\section{Sample collection and phenotypical identification}

A total of 50 fattening units located in Lombardy (Northern Italy) was selected, and
Correspondence: Ancuta Cezara Simon, Department of Food and Drug, University of Parma. Via del Taglio, 1043126 Parma, Italy. Tel: +39.0521.302754.

E-mail: ancutacezara.simon@studenti.unipr.it

Key words: MRSA; MLST; Spa-typing; pig chain; pigs

Contributions: The authors contributed equally.

Conflict of interests: The authors declare no conflict of interests.

Funding: This project has been chartered by the Italian Ministry of Health, project Classyfarm.

Received for publication: 18 July 2019.

Revision received: 4 October 2019.

Accepted for publication: 28 November 2019

This work is licensed under a Creative Commons Attribution-NonCommercial 4.0 International License (CC BY-NC 4.0).

C) Copyright: the Author(s), 2020

Licensee PAGEPress, Italy

Italian Journal of Food Safety 2020; 9:8412

doi:10.4081/ijfs.2020.8412

overall 400 samples were collected. In particular, dust samples were collected from 3 different sites on farm, i.e. barn, aeration device and feeder ( $\mathrm{n}=150$ samples), whereas cutaneous swabs were collected on the neck area from 5 animals in each farm at slaughterhouse right after stunning on the neck area $(\mathrm{n}=250$ samples $)$ following ISO 18593:2018 norm. All samples were stored at $4{ }^{\circ} \mathrm{C}$ and processed within $6 \mathrm{~h}$ at Istituto Zooprofilattico Sperimentale della Lombardia e dell'Emilia Romagna (IZSLER), Area of Diagnosis in Brescia.

Briefly, samples were enriched in Brain Hearth Infusion (BHI, Oxoid, Italy) containing $7.5 \% \mathrm{NaCl}$ and incubated for 18 $24 \mathrm{~h}$ at $37^{\circ} \mathrm{C}$. Next, $0,1 \mathrm{~mL}$ of the resulting broth was plated onto CHROMagar MRSA (bioMérieux, France) and Mannitol Salt Agar (MSA, Oxoid, Italy) as specified in ISO 6888-1:1999 norm. Suspected pink colonies grown on CHROMagar and yellow colonies surrounded by bright yellow zones on MSA were subjected to Gram staining, coagulase and urease tests. Phenotypical identification was performed by miniaturized biochemical system Api Staph (bioMérieux, France)

\section{Molecular characterization}

Detection of nuc, mecA and mecC (species confirmation and methicillin resistance)

The detection of nuc, mec A and mec $\mathrm{C}$ 
(mecA homologue) was carried out with a multiplex using specific primers as reported by Pichon et al. (2012). Briefly, the PCR reaction mix (final volume $20 \mu \mathrm{L}$ ) contained 1X HotStarTaq Master Mix (Qiagen INC, Hilden, Germany), $0.5 \mu \mathrm{M}$ of each primer, and $1 \mu \mathrm{L}$ DNA. The thermic profile was $95^{\circ} \mathrm{C}$ for $15 \mathrm{~min}$, followed by 35 cycles of $94^{\circ} \mathrm{C}$ for $30 \mathrm{~s}, 58^{\circ} \mathrm{C}$ for $40 \mathrm{~s}$, and $72^{\circ} \mathrm{C}$ for 1 min. The final elongation step was performed at $72^{\circ} \mathrm{C}$ for $10 \mathrm{~min}$. The amplified PCR products were distinguished by electrophoresis in a $2.5 \%$ agarose gel (Agarose Multi-Purpose, Roche) $120 \mathrm{~V}$ for 40 minutes stained with Eurosafe Nucleic Acid Stain (Euroclone, 1X). 100 bp DNA ladder (Invitrogen, $0.5 \mu \mathrm{g} / \mu \mathrm{L}$ ) was included.

For this work, twenty-five isolates identified as positive for nuc, mecA and $m e c \mathrm{C}$ genes, were processed for further analysis.

\section{Multilocus Sequence Typing (MLST) and staphylococcal protein A (spa)-typing}

MLST analysis was carried out as described by Enright et al. (2000). The Sequence Types (STs) were determined with the database available on the Staphylococcus aureus MLST website (https://pubmlst.org/ saureus) sited at the University of Oxford. For spa typing, the spa gene was amplified by PCR as described by Shopsin and colleagues (Shopsin et al., 1999) and spa types were determined with the Ridom StaphType software (Ridom GmbH, Würzburg, Germany). All DNA sequences were obtained with a $3500 x \mathrm{x}$ Genetic Analyzer (Applied Biosystems, Foster City, CA, USA).

Novel MLST and spa sequences were submitted to the respective database for the designation of the new profile.

\section{Phylogenetic analysis of the isolates}

The spa-typing data from MRSA isolates were analyzed by the BURP (Based Upon Repeat Pattern) algorithm, using the StaphType software v. 2.2.1 (Ridom GmbH, Germany). Neighbor-Joining tree of the isolates was constructed using MEGA 6 (Molecular Evolutionary Genetics Analysis; Tamura et al., 2013).

\section{Results}

Overall, 400 samples were collected, 150 from farm environment and 250 from animals at slaughterhouses. For the purposes of this work, a fattening unit was considered positive if MRSA were isolated at any stage from farm and/or from slaughterhouse, without considering the contamination source. A total of 17 out of 50 pig fattening units were positive for MRSA and overall 25 samples were positive for MRSA. At farm, 12 out of 250 samples were positive for MRSA (4,8\%), and 13 out of 150 samples at slaughter were identified as MRSA $(8,7$ $\%$, giving an overall incidence among samples of $6,25 \%(n=25)$.

The results of the MLST characterization allowed the identification of 5 different Sequence Types (STs). ST398 was the most frequent ST recovered among samples $(n=19)$, followed by ST97 $(n=3)$, ST4894 $(n=1)$ and ST30 (n=1) (Table 1). In addition, a new ST, namely ST5422, was detected in one isolate (Figure 1).

The isolates were also characterized by spa-typing, which allowed to identify 9 different profiles (Figure 2). Within the ST398, the most frequent spa-type detected was t899 $(n=6)$, followed by t011 $(n=5)$, t18498 $(n=4)$ and t1939 (n=2). Spa-types t318 and t034 were detected only in one sample each. Regarding ST97, two spa-types were identified: $\mathrm{t} 1730 \quad(\mathrm{n}=1)$ and $\mathrm{t} 4795$ $(n=2)$. Other subtypes identified among isolates were ST30/t318, ST4898/t18494, and ST5422/t1730.

Heterogeneity was observed in the spa profiles within fattening units. In fact, molecular characterization showed the presence of different spa-types and/or STs within the same farm in 6 out of 21 farms

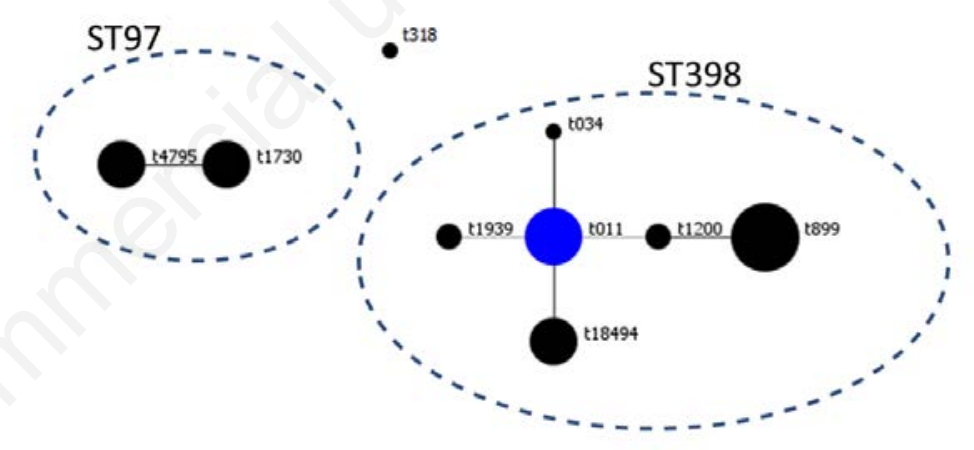

Figure 1. BURP clustering of spa types identified in this study. The spa typing data from S. aureus isolates were analyzed by the BURP algorithm (StaphType software v. 2.2.1, Ridom). Each spa type identified is depicted with a circle. The size of the circle is proportional to the frequency of the spa type in the population. Related spa types are connected with a black line. A blue circle corresponds to the clonal complex founder.

Table 1. Spa-types and MLST profiles of MRSA isolates.

\begin{tabular}{|c|c|c|c|c|}
\hline Spa-type & ST & $n$ farms where it was detected & $\%$ among positive farms & $\%$ farms surveyed \\
\hline t899 & 398 & 5 & 29 & 10 \\
\hline $\mathrm{t} 011$ & 398 & 5 & 29 & 10 \\
\hline t18494 & 398 & 3 & 18 & 6 \\
\hline $\mathrm{t} 1200$ & 398 & 2 & 12 & 4 \\
\hline t1939 & 398 & 2 & 12 & 4 \\
\hline $\mathrm{t} 4795$ & 97 & 1 & 6 & 2 \\
\hline $\mathrm{t} 1730$ & 97 & 1 & 6 & 2 \\
\hline $\mathrm{t} 1730$ & 5422 & 1 & 6 & 2 \\
\hline $\mathrm{t} 318$ & 30 & 1 & 6 & 2 \\
\hline t18494 & 4894 & 1 & 6 & 2 \\
\hline $\mathrm{t} 034$ & 398 & 1 & 6 & 2 \\
\hline
\end{tabular}


(Table 2). Results from BURP analysis showed that five farms were positive for MRSA presenting unrelated spa-types, whereas relatedness has been found among the strains isolated from the remaining unit (Figure 1, Table 2).

\section{Discussion}

Pig herds are an important reservoir for MRSA, with ST398 representing the most frequently reported subtype at European level in swine production chain (EFSA, 2009a; Battisti et al., 2010; Parisi et al., 2019). Although pig colonization with MRSA ST398 is frequent, infection in swine is rare. Nonetheless, it has been observed that such MRSA are able to colonize and cause infection in other species, including human, especially in areas with intense livestock-farming (Pan et al., 2009; Van Cleef et al., 2010). Although people professionally exposed to animals (owners, farmers, veterinarians and abattoir workers) are more likely to be colonized by MRSA ST398, colonization may also occur in family members not in direct contact with pigs (Cuny et al., 2009; Aires-de-Sousa, 2017).

In this work, it has been observed that the $42 \%$ of the sampled fattening units were positive for MRSA. Such prevalence is higher than the MRSA level in European production holdings reported by European Food Safety Authority (26.9\%; EFSA, 2009), but results are in line with previous findings that have reported different levels of MRSA prevalence in Italy, ranging from $33.9 \%$ to $64.7 \%$ (EFSA, 2009a; Parisi et al., 2019).

In order to investigate the genetic diversity of the MRSA isolates, molecular characterization by MLST and spa-typing was performed. Similarly to many other studies, the LA-MRSA ST398 was the ST most frequently recovered among pig farms (EFSA, 2009a; Battisti et al., 2010; Parisi et al., 2019). Within ST398, spa-typing revealed the presence of 6 different profiles, of which spa-type t899 was the most prevalent $(57.1 \%)$, which is the fifth most common genotype throughout Europe, following spa-types t011, t108, t034 and t127 (EFSA, 2009a). Among the other identified profiles, the spa-type t011, that is the most diffused in Europe (EFSA, 2009a), was found in the $23.8 \%$ of MRSA ST398. Spa-types t034 and t1939 were found in two and one isolates, respectively, in line with the European levels (EFSA, 2009a). To the best of authors' knowledge, spa-type t1200 has never been reported in scientific literature as recovered from pigs. Conversely, isolates presenting such spa-type have been collected from infection sites in hospital settings in Saudi Arabia, and thus t1200 is considered as HA lineage, raising concern for public health (Alkharsah et al., 2019). Finally, a new spa-type, namely t18494, has been found in three isolates belonging to ST398. This profile was also identified in one other isolate carrying ST4894.

Two spa-types belonging to the LA-
MRSA lineage ST97 (i.e., t1730 and t4795) were recovered in three samples from two different farms. Both spa-types were previously reported by Locatelli et al. (2017) in samples collected from dairy cattle herds, swine farms related to dairy herds, and humans in contact with herds, suggesting that these genotypes could be transmitted from animals to humans and vice versa. Furthermore, an isolate belonging to ST30/t318 was reported for the first time in Italian pig farms with this work, although ST30 carrying other spa-types (i.e., t012 and t093) were isolated from swabs collected in slaughterhouses located in Southern Italy (Normanno et al., 2015). Isolates belonging to ST30 have been described as CA-MRSA; in particular, ST30/t318 has been reported to be responsible for abscesses, bloodstream or necrotic infections in different parts of the world (e.g., Czech Republic, China) (Jiang et al., 2013; Rájová et al., 2016), and the presence of an isolate collected from farm

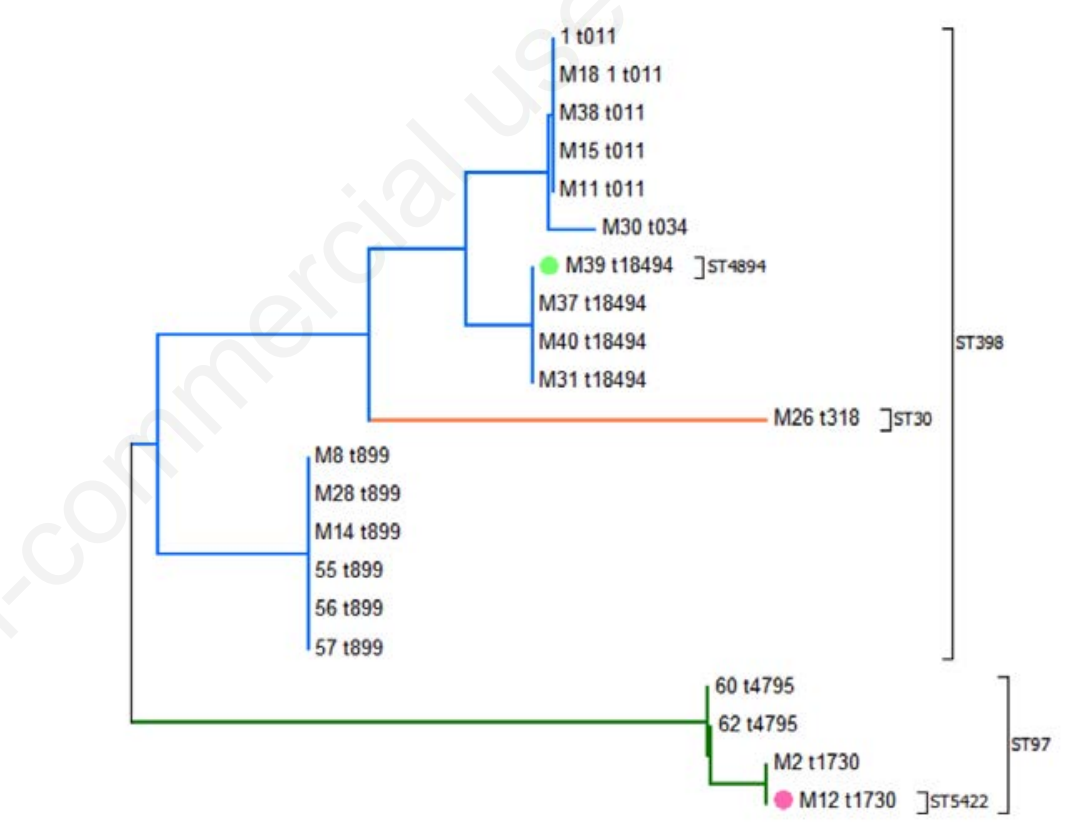

Figure 2. Neighbor-Joining tree of the isolates included in this study. Different colors correspond to the Sequence Types (STs). The analyses were conducted in MEGA6 (Tamura et al., 2013).

Table 2. Molecular pattern distribution among farms with more than one spa-type or ST detected.

\begin{tabular}{lcccc}
\hline Farm code & $n$ spa-types & spa-types & STIs \\
1 & 2 & $\mathrm{t} 1730, \mathrm{t} 899$ & unrelated & 5422,398 \\
2 & 2 & $\mathrm{t} 1939, \mathrm{t} 034$ & unrelated & 398 \\
\hline 3 & 2 & $\mathrm{t} 18494, \mathrm{t} 011$ & related & 398 \\
4 & 2 & $\mathrm{t} 1730, \mathrm{t} 011$ & unrelated & 97,398 \\
\hline 5 & 2 & $\mathrm{t} 18494, \mathrm{t} 1200$ & unrelated & 398 \\
6 & 1 & $\mathrm{t} 18494$ & unrelated & 398,4894 \\
\hline
\end{tabular}


belonging to this lineage is of concern for the health of people coming into contact with animals. Finally, the molecular characterization allowed to identify an isolate belonging to a novel ST, further characterized as ST5422, showing spa-type t1730. The presence of different spa-types and/or STs within the same pig farm was observed in 6 out of 50 fattening units (Table 2). Unrelated spa-types (up to three spatypes) either belonging to ST398, ST97, ST4894 or ST5422 have been found within a same farm (Figure 1, Table 2).

It is not clear what the presence of different genotypes in a fattening unit is related to, although in other works it has been found that genetic variability was due to mutations in the strains already present in the farm (e.g. deletion of one repeat in spatype $\mathrm{t} 18498$ results in spa type $\mathrm{t} 034$, and the deletion of one repeat in 1899 results in t1939) or to the introduction of new strains by other animals or humans visiting the farms (van Duijkeren et al., 2008; Verhegghe et al., 2013). The presence of different MRSA genotypes within farms could favor the exchange of virulence determinants among different lineages (van Duijkeren et al., 2008; Verhegghe et al., 2013), with potential implications for both animal and human health.

The reduction of MRSA animal-human transmission is possible through the use and the implementation of management practices aimed at increasing on-farm biosecurity. Also the adoption and the improvement of measures such as good husbandry practices, Good Hygiene Practices (GHP) and Good Manufacturing Practices (GMP) on farms, in slaughterhouses and in food production areas are useful in controlling the spread of bacteria (EFSA, 2009b), leading to a reduction of related health risks and to a higher microbiological quality and safety of swine products. In fact, a serious concern is related to the occurrence of foodborne transmission of MRSA, since MRSA presence in pork meat and meat products has been reported by different authors (Petternel et al., 2014; Tang et al., 2017). The ST398 lineage is the most commonly found among the foodborne-associated MRSA, including some of the genotypes identified in this work, such as the spa-types t011 and t034 (EFSA, 2009b; Petternel et al., 2014; Tang et al., 2017).

These findings stress once more that practical measures should be taken within pig farms to reduce the spread of MRSA.

\section{Conclusions}

Although ST398 colonization in humans is mostly related to professional exposure, in some cases it can cause severe infections
(Pan et al., 2009). Furthermore, other molecular profiles have been found among the isolates, such as the CA-MRSA ST30, that is also responsible for different infection in humans. The results of this work highlight the necessity of monitoring both the community and the animal reservoirs, and of adopting and implementing measures able to control the spread of MRSA among animals, in order to reduce zoonotic transmission of this pathogen by direct or indirect humananimal contact and through the consumption of contaminated foodstuffs.

\section{References}

Abdelbary MMH, Basset P, Blanc DS, Feil EJ, 2017. The Evolution and Dynamics of Methicillin-Resistant Staphylococcus aureus. In Genetics and Evolution of Infectious Diseases: Second Edition.

Aires-de-Sousa M, 2017. Methicillinresistant Staphylococcus aureus among animals: current overview. Clin Microbiol Infect 23:373-80.

Alkharsah KR, Rehman S, Alnimr A, Diab A, Hawwari A, Tokajian S, 2019. Molecular typing of MRSA isolates by spa and PFGE. J King Saud Univ - Sci

Battisti A, Franco A, Merialdi G, Hasman H, Iurescia M, Lorenzetti R, Feltrin F, Zini M, Aarestrup FM, 2010. Heterogeneity among methicillin-resistant Staphylococcus aureus from Italian pig fattening holdings. Vet Microbiol 142:361-6.

Cuny C, Nathaus R, Layer F, Strommenger B, Altmann D, Witte W, 2009. Nasal Colonization of Humans with Methicillin-Resistant Staphylococcus aureus (MRSA) CC398 with and without Exposure to Pigs. PLoS One 4:e6800.

Doulgeraki AI, Di Ciccio PA, Ianieri A, Nychas G-JE, 2016. Methicillin-resistant food-related Staphylococcus aureus: a review of current knowledge and biofilm formation for future studies and applications. Res Microbiol 168:1-15.

European Food Safety Authority (EFSA), 2009a. Analysis of the baseline survey on the prevalence of methicillin-resistant Staphylococcus aureus (MRSA) in holdings with breeding pigs, in the EU, 2008 - Part A: MRSA prevalence estimates. EFSA J 7:1376.

European Food Safety Authority (EFSA), 2009b. Assessment of the Public Health significance of methicillin resistant Staphylococcus aureus (MRSA) in animals and foods. EFSA J 7:993.

Enright MC, Day NPJ, Davies CE, Peacock SJ, Spratt BG, 2000. Multilocus sequence typing for characterization of methicillin-resistant and methicillinsusceptible clones of Staphylococcus aureus. J Clin Microbiol 38:1008-15.

Jiang W, Zhou Z, Zhang K, Yu Y, 2013. Epidemiological investigation of community-acquired Staphylococcus aureus infection. Genet Mol Res 12:6923-6930.

Lassok B, Tenhagen B-A, 2013. From pig to pork: Methicillin-resistant Staphylococcus aureus in the pork production chain. J Food Prot 76:1095108.

Locatelli C, Cremonesi P, Caprioli A, Carfora V, Ianzano A, Barberio A, Morandi S, Casula A, Castiglioni B, Bronzo V, Moroni P, 2017. Occurrence of methicillin-resistant Staphylococcus aureus in dairy cattle herds, related swine farms, and humans in contact with herds. J Dairy Sci 100:608-19.

Normanno G, Dambrosio A, Lorusso V, Samoilis G, Di Taranto P, Parisi A, 2015. Methicillin-resistant Staphylococcus aureus (MRSA) in slaughtered pigs and abattoir workers in Italy. Food Microbiol 51:51-6.

Oniciuc, E. A., Nicolau, A. I., Hernández, M., Rodríguez-Lázaro, D. (2017). Presence of methicillin-resistant Staphylococcus aureus in the food chain. Trends Food Sci Technol 61:49-59.

Pan A, Battisti A, Zoncada A, Bernieri F, Boldini M, Franco A, Giorgi M, Iurescia M, Lorenzotti S, Martinotti M, Monaci M, Pantosti A, 2009. Communityacquired methicillin-resistant Staphylococcus aureus ST398 infection, Italy. Emerg Infect Dis 15:845-7.

Parisi A, Caruso M, Normanno G, Latorre L, Miccolupo A, Fraccalvieri R, Intini F, Manginelli T, Santagada G, 2019. MRSA in swine, farmers and abattoir workers in Southern Italy. Food Microbiol 82:287-93.

Petternel C, Galler H, Zarfel G, Luxner J, Haas D, Grisold AJ, Reinthaler FF, Feierl G, 2014. Isolation and characterization of multidrug-resistant bacteria from minced meat in Austria. Food Microbiol 44:41-6.

Pichon B, Hill R, Laurent F, Larsen AR, Skov RL, Holmes M, Edwards GF, Teale C, Kearns AM, 2012. Development of a real-time quadruplex PCR assay for simultaneous detection of nuc, pantonvalentine leucocidin (PVL), mecA and homologue mec $\mathrm{A}_{\mathrm{LGA} 251}$. J Antimicrob Chemother 67:2338-41.

Rájová J, Pantůček R, Petráš P, Varbanovová I, Mašlaňová I, Beneš J, 2016. Necrotizing pneumonia due to clonally diverse Staphylococcus aureus strains 
producing Panton-Valentine leukocidin: the Czech experience. Epidemiol Infect 144:507-15.

Sieber RN, Skov RL, Nielsen J, Schulz J, Price LB, Larsen AR, Stegger M, 2018. Drivers and Dynamics of MethicillinResistant Livestock- Associated Staphylococcus aureus CC398 in Pigs and Humans in Denmark. mBio 9:e1242-18

Shopsin B, Gomez M, Montgomery SO, Smith DH, Waddington M, Dodge DE, Bost DA, Riehman M, Naidich S, Kreiswirth BN, 1999. Evaluation of protein A gene polymorphic region DNA sequencing for typing of Staphylococcus aureus strains. J Clin Microbiol 37:3556-63.

Tamura K, Stecher G, Peterson D, Filipski A, Kumar S, 2013. MEGA6: Molecular Evolutionary Genetics Analysis version 6.0. Mol Biol Evol 30:2725-9.

Tang Y, Larsen J, Kjeldgaard J, Andersen PS, Skov R, Ingmer H, 2017. Methicillinresistant and -susceptible Staphylococcus aureus from retail meat in Denmark. Int $\mathrm{J}$ Food Microbiol 249:72-6.

Taylor AR, 2013. Methicillin-resistant Staphylococcus aureus infections. Prim Care - Clin Off Pract 40:637-54.

Van den Broek IVF, Van Cleef BAGL, Haenen A, Broens EM, Van der Wolf PJ, Van den Broek MJM, Huijsdens XW, Kluytmans JAJW, Van de Giessen AW, Tiemersma EW, 2009. Methicillinresistant Staphylococcus aureus in people living and working in pig farms. Epidemiol Infect 137:700-8.

Van Cleef BAGL, Broens EMEM, Voss A, Huijsdens XW, Züchner L, Van Benthem BHB, Kluytmans JAJW, Mulders MN, Van De Giessen AW, 2010. High prevalence of nasal MRSA carriage in slaughterhouse workers in contact with live pigs in The Netherlands. Epidemiol Infect 138:756-63.

Van Duijkeren E, Ikawaty R, BroekhuizenStins MJ, Jansen MD, Spalburg EC, Neeling AJ de, Allaart JG, van Nes A, Wagenaar JA, Fluit AC, 2008. Transmission of methicillin-resistant
Staphylococcus aureus strains between different kinds of pig farms. Vet Microbiol 126:383-9.

Verhegghe M, Pletinckx LJ, Crombé F, Vandersmissen T, Haesebrouck F, Butaye P, Heyndrickx M, Rasschaert G, 2013. Methicillin-resistant Staphylococcus aureus (MRSA) ST398 in pig farms and multispecies farms. Zoonoses Public Health 60:366-74.

Zarazaga M, Gómez P, Ceballos S, Torres C, 2017. Molecular epidemiology of Staphylococcus aureus lineages in the animal-human interface. In: Fetsch A., ed. Staphylococcus aureus. Academic Press, Cambridge, MA, pp. 189-214.

Zehra A, Gulzar M, Singh R, Kaur S, Gill JPS, 2019. Prevalence, multidrug resistance and molecular typing of methicillin-resistant Staphylococcus aureus (MRSA) in retail meat from Punjab, India. J Glob Antimicrob Resist 16:152-8. 